\title{
Is Hospital Discharge the Rube Goldberg Machine of Academic Internal Medicine?
}

\author{
Frances Mao, MD*, S. Ryan Greysen, MD, MHS, MA ${ }^{1,2}$
}

'Section of Hospital Medicine, Division of General Internal Medicine, Perelman School of Medicine at the University of Pennsylvania, Philadelphia, Pennsylvania; ${ }^{2}$ Leonard Davis Institute of Health Economics, The Wharton School at the University of Pennsylvania, Philadelphia, Pennsylvania.

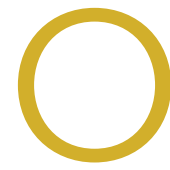

ne of the least taught yet most complicated tasks confronting new trainees is the bewildering process of discharging a patient. On an internal medicine service, this process can often resemble a Rube Goldberg machine, in which a "simple" task is accomplished through a series of interconnected, almost comically convoluted, yet separate steps that are triggered one after another and must be executed perfectly in sequence for success. It seems easy at first; just tap out a few sentences in the discharge paperwork, do a quick medication reconciliation, and a click of a button later, voila! The patient magically falls off the list and is on their merry way home. In reality, it only takes one wrench thrown into the Rube Goldberg machine to take down the whole operation. Much to the chagrin of internal medicine interns across the country, residents quickly learn that discharge planning is usually far from straightforward and that a myriad of obstacles (often dynamic and frustratingly unpredictable) can stand in the way of a successful discharge.

While some surgical services can streamline discharge processes to target defined lengths of stay based on a particular diagnosis, general medicine patients tend to have greater numbers of comorbid conditions, complex hospital courses, and wider variation in access to posthospital healthcare. In addition, there is very little formal instruction in transitions of care, and most residents identify direct patient care (learning by doing) as the primary mode of education. ${ }^{1,2}$ Struggling through the process of finding an appropriate placement, ensuring adequate outpatient follow-up, and untangling a jumbled mess of a medication reconciliation is often the only way that housestaff learn the Sisyphean task of transitioning care out of the hospital. The unpredictability and intensity of patient care adds to the ever growing list of competing demands on the time and attention of residents. Attendings face pressure on all sides to not only provide exemplary patient care and an educational experience but also to optimize hospital throughput by discharging patients as soon as possible (and ideally before noon). No wonder that the discharge

\footnotetext{
*Address for correspondence: Frances Mao, MD, Section of Hospital Medicine, Division of General Internal Medicine, Hospital of the University of Pennsylvania - Maloney Building, Suite 5032, 3400 Spruce Street, Philadelphia, PA 19104; Telephone: 267-324-6236; Fax: 215-662-6250; E-mail: frances.mao@ uphs.upenn.edu
}

Received: October 1, 2013; Accepted: October 3, 2018

๑ 2018 Society of Hospital Medicine DOI 10.12788/jhm.3116

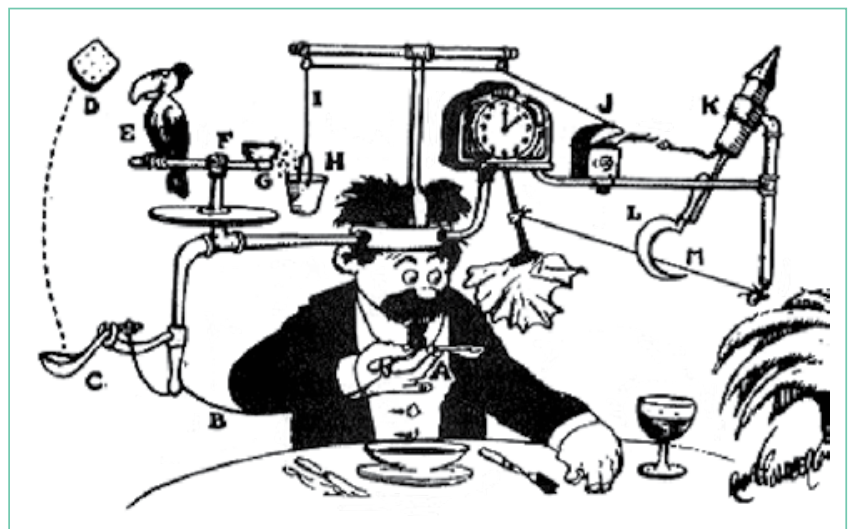

Professor Butts and the Self-Operating Napkin (1931). Soup spoon (A) is raised to mouth, pulling string (B) and thereby jerking ladle (C), which throws cracker (D) past parrot (E). Parrot jumps after cracker and perch (F) tilts, upsetting seeds $(G)$ into pail $(H)$. Extra weight in pail pulls cord $(I)$, which opens and ignites lighter $(J)$, setting off skyrocket $(K)$, which causes sickle $(L)$ to cut string $(M)$, allowing pendulum with attached napkin to swing back and forth, thereby wiping chin. Rube Goldberg - Originally published in Collier's, September 261931.

process can threaten to unravel at any time, with delays and complications in discharge metamorphosing into increased length of stay (LOS), poorer outcomes, and increased 30-day readmission rates. As on-the-ground providers, what realities do we face when challenging ourselves to discharge patients before noon, and what practical changes in our workflow can we make to reach this goal?

In this month's Journal of Hospital Medicine, Zoucha et al. examine these questions in real time by identifying barriers preventing both "definite" and "possible" discharges at three representative time points over the course of randomly chosen weekdays. They surveyed both housestaff and attendings at five academic hospitals across the United States, and the majority of patients were cared for on teaching services. ${ }^{3}$ Reflecting the inherent differences in workflow between teaching and nonteaching services, delays in definite discharges on teaching services were most often hindered by completing rounds and the need to staff the patient with the attending, whereas nonresident services identified other patient-care-related (both urgent and nonurgent) issues to be the culprits. Late-afternoon discharges were delayed on teaching services due to outstanding paperwork and follow-up arrangements, both of which most senior residents are keenly aware of and make their best effort to complete ahead of time. Patients designated as "possible" discharges were awaiting clinical improvement and resolution of disposition issues dependent on social work 
and safe placement, which reasonably seemed independent of service type. These descriptive findings suggest that nonresident services are more efficient than resident teams, and we are keen to identify novel solutions, such as dedicated discharge coordinators, ${ }^{4}$ to facilitate the discharge process on resident teams without detracting from the educational value of the rotation.

Zoucha et al. also found that factors beyond our control (having a lower daily census, attending on a nonresident service) were significantly associated with both earlier discharge order entry times and the actual time of patient discharge. ${ }^{3}$ While it is tempting to foist the entirety of the blame on extrinsic factors such as discharge placement and insurance issues, the reality is there might be some workflow changes that could expedite the discharge process. The authors are correct to emphasize that rounding style, in which discharges are prioritized to be seen first, is a behavior modification worth targeting. The percentage of teams that routinely see discharges first is not well studied, as other factors, such as clinically unstable patients, new admissions from overnight, and even mundane characteristics such as geographic location in the hospital, can also compete for prioritization in rounding order. Given the authors' findings, we are eager to see further work in this area as prioritization of discharges during rounds could conceivably be studied within the context of a randomized controlled trial. Other innovations in rounding styles such as rounding-in-flow ${ }^{5}$ (in which all tasks are completed for a single patient before rounding on the next patient) can also significantly reduce the time to discharge order placement.

With help from the Penn Medicine Center for Health Care Innovation, we are actively studying bottlenecks in the discharge process by developing an interactive platform focused on delivering real-time information to all members of the healthcare team. Rapid rounds are held every morning with the attending physician, floor nursing leadership, physical therapy, social worker, and case management to quickly identify pending tasks, anticipated disposition, and a target date of discharge. Efficiency is key, as each team is limited to approximately 5-10 minutes. Previous studies (mostly pre-post studies) have shown that this simple intervention significantly reduced LOS, ${ }^{6,7}$ increased rates of discharge before noon, ${ }^{8}$ and was improved by electronic tracking tools. ${ }^{9}$ Our multidisciplinary rounds are unique in that information is then entered into an intuitive, web-based platform, which allows consolidation and analysis that permits generation of real-time statistics. By standardizing the discharge planning process, we hope to streamline a previously fragmented process and maximize the efficiency of hospital resource utilization.

Ultimately, high-quality care of complex patients on internal medicine services from admission to discharge requires hard work, smart utilization of resources, and a little bit of luck. There may not be a secret ingredient that guarantees perfectly efficient discharges $100 \%$ of the time, but this study inspires us to ponder additional approaches to this longstanding problem. The authors are to be congratulated for a rigorous study that illuminates where we as healthcare providers are able to realistically intervene to expedite the discharge process. First, having a lower census cap may not be possible in this era of maximal hospital usage, but this work suggests that thoughtful management of time on rounds may be a way to address the underlying problem. Secondly, the superior efficiency of nonteaching services may merely reflect the increased experience of the providers, and a realistic solution could be to implement a formal curriculum to educate housestaff about the discharge process, which would simultaneously address residency competency standards for transitions of care. Finally, the role of innovative informatics tools will surely open further avenues of investigation, as we continually evolve in response to intensifying standards of modern, efficient healthcare delivery in the $21^{\text {st }}$ century. It may not be possible to eliminate the complexity from this particular Rube Goldberg machine, but taking the steps above may allow us to implement as many fail-safes as we can.

Disclosures: The authors have nothing to disclose.

\section{References}

1. Young E, Stickrath C, McNulty M, et al. Residents' exposure to educational experiences in facilitating hospital discharges. J Grad Med Educ. 2017;9(2):184-189. doi: 10.4300/JGME-D-16-00503.1.

2. Greysen SR, Schiliro D, Curry L, et al. "Learning by doing" - Resident perspectives on developing competency in high-quality discharge care. J Gen Intern Med. 2012;27(9):1188-1194. doi: 10.1007/s11606-012-2094-5.

3. Zoucha J, Hull M, Keniston A, et al. Barriers to Early Hospital Discharge: A Cross-Sectional Study at Five Academic Hospitals. J Hosp Med. 2018;13(12):816-822. doi: 10.12788/jhm.3074.

4. Finn KM, Heffner R, Chang Y, et al. Improving the discharge process by embedding a discharge facilitator in a resident team. J Hosp Med. 2011;6(9):494500. doi: 10.1002/jhm.924.

5. Calderon AS, Blackmore CC, Williams BL, et al. Transforming ward rounds through rounding-in-flow. J Grad Med Educ. 2014;6(4):750-755. doi: 10.4300/ JGME-D-13-00324.1.

6. Kane M, Rohatgi N, Heidenreich PA, et al. Lean-based redesign of multidisciplinary rounds on general medicine service. J Hosp Med. 2018;13(7):482-485. doi: $10.12788 / \mathrm{jhm} .2908$.

7. Gonçalves-Bradley D, Lannin N, Clemson L, Cameron ID, Shepperd S. Discharge planning from hospital. Cochrane Database Syst Rev. 2016;1-3. doi: 10.1002/14651858.CD000313.pub5.www.cochranelibrary.com.

8. Wertheimer B, Jacobs REA, Bailey $M$, et al. Discharge before noon: an achievable hospital goal. J Hosp Med. 2014;9(4):210-214. doi: 10.1002/ jhm.2154.

9. Meo N, Paul E, Wilson C, Powers J, Magbual M, Miles KM. Introducing an electronic tracking tool into daily multidisciplinary discharge rounds on a medicine service: a quality improvement project to reduce length of stay. BMJ Open Qual. 2018;7(3):e000174. doi: 10.1136/bmjoq-2017-000174. 